\title{
Dual-Band Tunable Recursive Active Filter
}

\author{
Daniel Segovia-Vargas, Member, IEEE, Óscar García-Pérez, Vicente González-Posadas, and
} Francisco Aznar-Ballesta, Member, IEEE

\begin{abstract}
This letter presents a novel recursive active filter topology that provides dual-band performance, with independent tuning capability in both bands. The dual-band operation is achieved by using two independent feedback lines. Additionally, linear phase shifters based on left-handed cells are included in these two branches in order to tune the center frequency of both pass bands.
\end{abstract}

Index Terms-Active filters, dual-band circuits, left handed (LH) transmission lines, tunable filters.

\section{INTRODUCTION}

$\mathbf{M}$ ICROWAVE active filters are of great interest in many modern communication systems, since they can provide more compact size, higher gain and higher selectivity than current passive resonators. As a counterpart, some additional considerations such as power consumption, stability or noise figure should be considered. During the last years several active filter approaches have been developed. Recursive active filters are topologies in which several samples of the input signal are weighted and delayed along several paths, and are finally properly combined at the output [1]. This topology presents several advantages, such as simple design and high gain. Additionally, it can be easily implemented in both discrete [1] and monolithic (MMIC) [2] technologies.

A natural step in active filters is obtaining a voltage-controlled frequency-tunable circuit, since voltage supply is already present in the structure. Thus, it improves the versatility of the filter to be used in any general-purpose application. Tuning is particularly simple to obtain in first-order recursive structures, just by including a phase shifter in the feedback line. It allows to obtain a very linear response of the center frequency with respect to the phase shift. Thus, several single-band tunable recursive active filters have been proposed in the literature [3]

The rapid emergence of wireless devices simultaneously supporting several communication standards makes very useful the implementation of multiband microwave components. Most effort in multiband filters has been focused in the design of passive resonators. First advances in multiband active filters have been recently presented in [4], [5]. These novel dual-band active filters combine recursive topologies and composite right/left-handed transmission lines (CRLH-TLs) [6]. By using

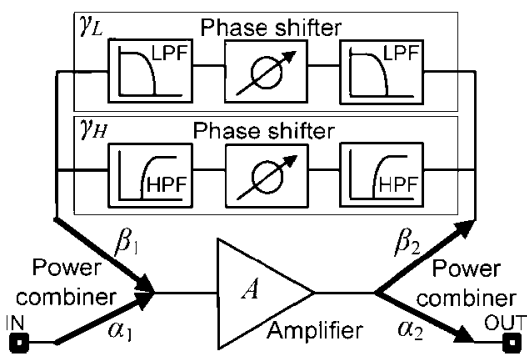

Fig. 1. General schematic of the proposed dual-band tunable active filter

a CRLH-TL to feed back the signal, and taking advantage of its inherent non-linear phase response, it is possible to obtain two pass bands centered at two arbitrary frequencies.

Future telecommunication systems, such as cognitive radio devices, will require multipurpose and highly versatile radio frequency components. Thus, combining both multi-frequency and tuning capabilities in active filter designs would be a very adequate solution for this type of applications. However, no active filter design with several tunable bands has been presented before. One of the main features of the solutions presented in [4], [5] is that a single feedback line is used to provide the dual-band performance. However it presents a constraint for tuning, since any phase shift in this feedback line would affect both frequency bands.

This letter presents a novel approach in which a recursive topology with two feedback lines is used to provide dual-band performance with independent tuning capability in the two pass bands. Highly linear phase shifters based on left-handed cells have been included in both branches [7]. They allow a quite linear response of the center frequencies with respect to the corresponding control voltages.

\section{DUAL-BAND TUNABLE ACTIVE FILTERS}

The basic scheme of the proposed dual-band tunable active filter is shown in Fig. 1. Two power combiners are used to combine/split the signals at the input/output. They provide isolation between the terminal ports and the feedback line, and therefore, avoid stability problems. There is a direct branch in which the gain block is placed. It must be remarked that a single amplifier is used to provide the multiband operation, which is a great advantage to minimize the power consumption. Finally, there are two feedback lines to provide the desired dual-band performance. Each feedback structure operates at low and high frequencies respectively, and they are isolated by means of low/ high-pass filters. Attending to the basic operation of first-order recursive filters, the pass bands are obtained at the frequencies at which the sum of phases in the loop is multiple of $2 \pi$ [4]. In this case it corresponds to

$$
\begin{aligned}
\angle A\left(f_{L}\right)+\angle \gamma_{L}\left(f_{L}\right)+\angle \beta_{1}\left(f_{L}\right)+\angle \beta_{2}\left(f_{L}\right) & =m 2 \pi \\
\angle A\left(f_{H}\right)+\angle \gamma_{H}\left(f_{H}\right)+\angle \beta_{1}\left(f_{H}\right)+\angle \beta_{2}\left(f_{H}\right) & =n 2 \pi
\end{aligned}
$$




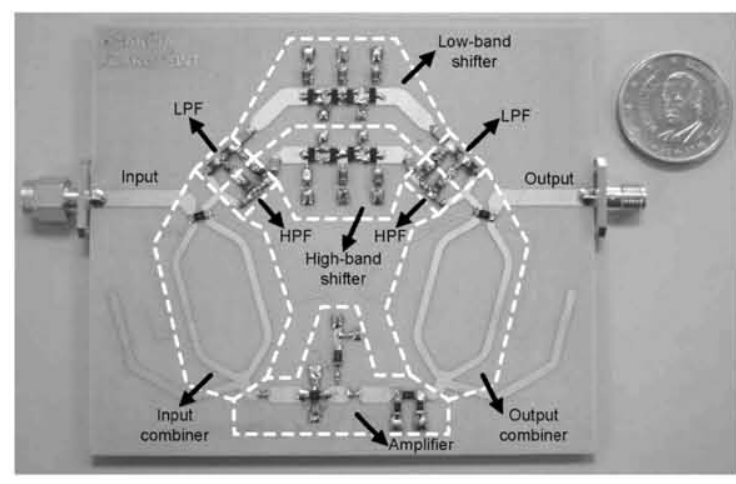

Fig. 2. Photograph of the manufactured dual-band tunable filter.

where $\angle$ denotes the angle, $A, \gamma_{L / H}$ and $\beta_{1 / 2}$ are the transmission parameters of the amplifier, the feedback lines and the upper branches of the combiner, $m$ and $n$ are integer numbers and $f_{L}$ and $f_{H}$ are the lower and higher band-pass frequencies. It can be seen how in the lower band, the higher-band feedback line is "invisible" and the pass-band is dependent on $\gamma_{L}$, and it occurs the opposite in the higher band. In this case, the phase of $\gamma_{L}$ and $\gamma_{H}$ will be tunable. The transfer function of the filter $H(f)$ can be obtained as [5]

$$
H(f)=\frac{A \alpha_{1} \alpha_{2}}{1-\beta_{1} \beta_{2} \gamma_{L / H} A}
$$

where $\alpha_{1 / 2}$ are the transmission parameters of the lower branches of the combiners. On the other hand, the noise factor of the filter $F_{f}$ can be obtained as [6]

$$
F_{f}=1+\frac{1}{\alpha_{1}^{2}}\left(F_{A}-1\right)+\left(\frac{\beta_{2}-\beta_{1} A \gamma_{L / H}}{\alpha_{1} \alpha_{2} A}\right)
$$

where $F_{A}$ is the noise factor of the amplifier.

Although a dual-band implementation is demonstrated in this letter, the proposed idea can be reutilized to design filters with arbitrary number of tunable sub-bands, just by including more feedback lines and taking into account some considerations. Firstly, low/high-pass filters should be replaced by band-pass filters, isolating the feedback lines in their corresponding frequency ranges. Additionally, the power combiners and the gain block should cover the different frequency regions at which the filter operates (i.e.,: multiband or wideband solutions).

\section{EXPERIMENTAL RESULTS}

In order to validate the theoretical principles presented in the previous section, a dual-band tunable active filter working in bands around 765 and $1680 \mathrm{MHz}$ has been designed and measured. The circuit board and the schematic are shown in Figs. 2 and 3. The gain block has been implemented by cascading a commercial amplifier (ERA-5+ from Minicircuits) and a resistive attenuator to limit the gain and avoid instabilities [4]. The measured gain of this block is around $4.9 \mathrm{~dB}$ and $4.7 \mathrm{~dB}$, and the noise figure is around 3.5 and $3.8 \mathrm{~dB}$ in lower and higher bands respectively. The low/high-pass filters have been implemented by two cascaded LC sections. The rejection of the undesired frequency range is better than $24 \mathrm{~dB}$ and $21 \mathrm{~dB}$, respectively. The power combiners are dual-band Wilkinson hybrids, designed by means of stub-loaded $90^{\circ}$ transmission lines [8]. As

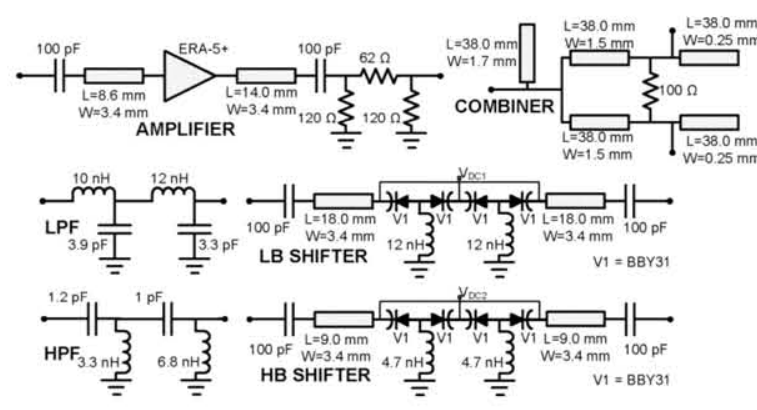

Fig. 3. Circuit schematic of the different parts of the manufactured filter.

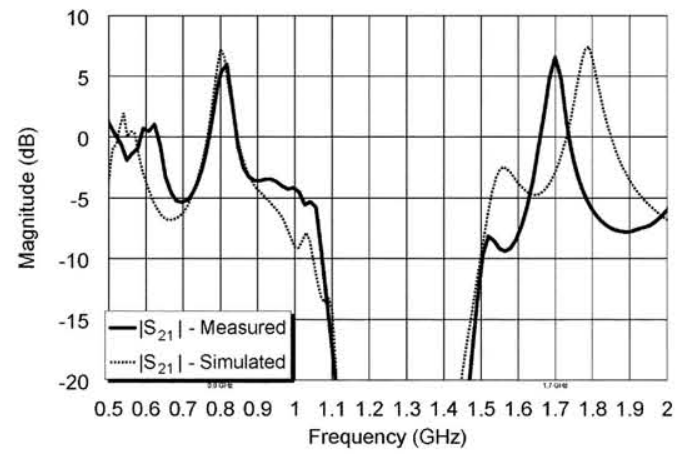

Fig. 4. Transmission coefficient of the active filter for $V_{D C 1}=V_{D C 2}=$ $18 \mathrm{~V}$.

it was shown in [4], this type of combiners allows improving the rejection at intermediate frequencies. A Wilkinson design has been chosen instead of others (e.g.,: branch-line) because of its broader bandwidth and its flatter transmission response, which is very useful to obtain a response with almost constant gain during the tuning. Finally, the phase shifters are designed following the topology presented in [7]. This phase shifter design is based on left handed cells (i.e.,: series capacitors and shunt inductors) and is inspired on the model of a metamaterial transmission line [6]. In this case, two T-cells have been used, and the variable capacitance is obtained from varactor diodes (model BBY-31 from Philips). Additionally, two transmission line sections are included in the feedback structure in order to center the tunable band at the desired value. The simulated insertion losses are lower than 1.5 and $2.2 \mathrm{~dB}$ in the lower and higher frequency ranges. Two control voltages $\left(V_{D C 1}\right.$ and $\left.V_{D C 2}\right)$ are used to independently control the center frequency of both low/high frequency pass bands respectively.

The measured filter response for a fixed value of the control voltages is shown in Fig. 4. There is a good agreement with the simulated response. A necessary condition for stability is ensure that the lop gain remains below than $0 \mathrm{~dB}$ [4]. Fig. 5 shows that this condition is fulfilled in the in-band and out-of-band regions. Additionally, the reflection coefficients are below $0 \mathrm{~dB}$ in that frequency range. The measured filter response in the two bands for different values of the corresponding control voltages is shown in Figs. 6 and 7. The tunable frequency range with less than $3 \mathrm{~dB}$ gain ripple covers a range of $312 \mathrm{MHz}$ around 765 $\mathrm{MHz}$ in the lower band, and covers $100 \mathrm{MHz}$ around $1680 \mathrm{MHz}$. The higher frequency band has been shifted around $100 \mathrm{MHz}$ nearer to the notch with respect to the simulation, so it has been attenuated in the lower part. It should be noticed that the gain of this filter topology is very sensitive with variations in the transmission in the loop [4], which makes difficult to achieve 


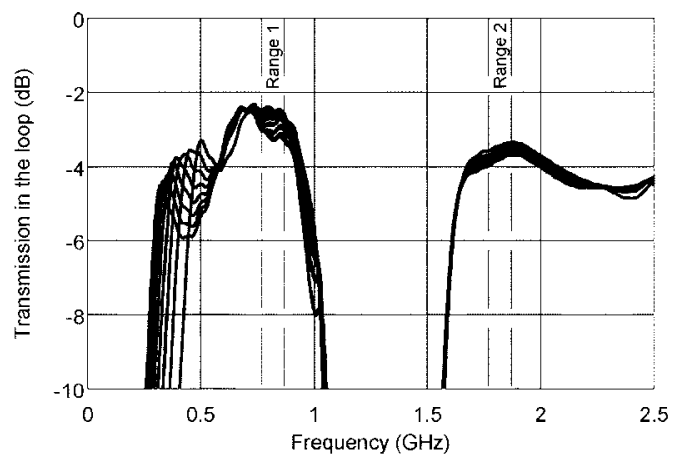

Fig. 5. Transmission coefficient in the loop for $V_{D C 1}=0-26 \mathrm{~V}, V_{D C 2}=$ $0-26 \mathrm{~V}$.

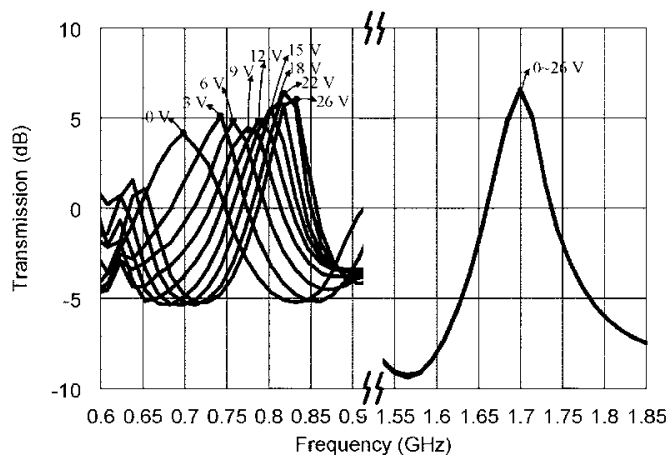

Fig. 6. Measured transmission coefficients for a variable value of $V_{D C_{1}}$ (specified over the curves), and a fixed value of $V_{D C 2}=18 \mathrm{~V}$.

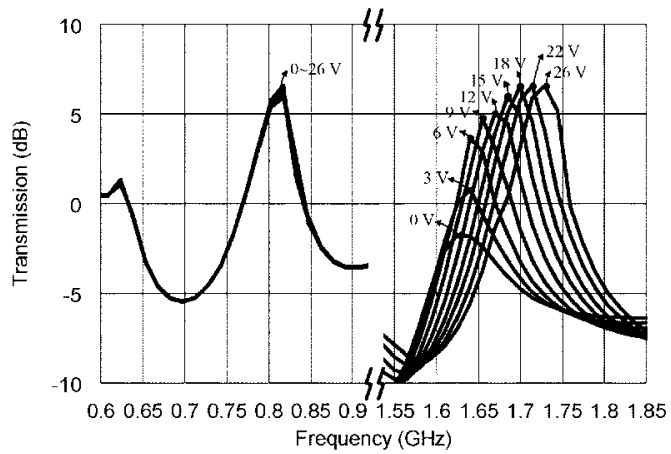

Fig. 7. Measured transmission coefficients for a fixed value of $V_{D C 1}=18 \mathrm{~V}$, and a variable value of $V_{D C 2}$ (specified over the curves).

very flat responses. Some spurious peaks appear in the response as undesired solutions of (1) [4]. They could be eliminated by adding additional filtering or by using broadband combiners [5]. Broader tuning regions can be obtained by increasing the number of cells of the shifters. The typical $3 \mathrm{~dB}$ bandwidths of the different bands are around $40 \mathrm{MHz}$. The curves of the center frequency as a function of the control voltages are almost linear for most the voltage range, as shown in Fig. 8. The in-band reflection coefficients are better than $-6 \mathrm{~dB}$ in all the cases. The typical noise figure is around $7.2 \mathrm{~dB}$ and $8.0 \mathrm{~dB}$ in both bands. It should be noticed that half-power combiners increment the noise of the filter more than $3 \mathrm{~dB}$ with respect to the isolated gain block. The noise figure can be improved by using asymmetric combiners [2]. Finally, the main results are summarized in Table $\mathbf{I}$.

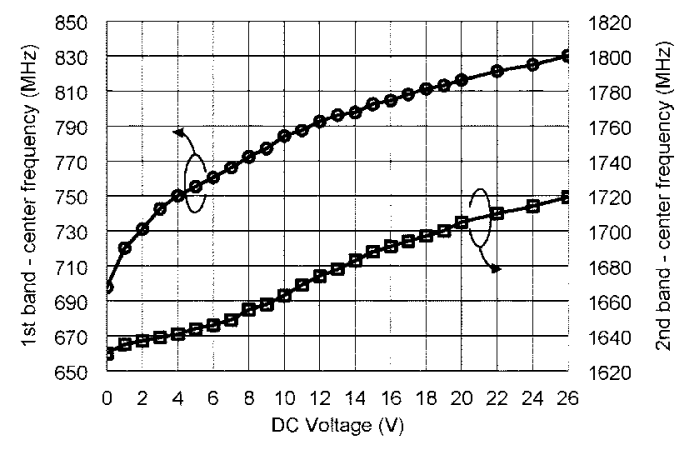

Fig. 8. Variation of the center frequency as a function of the control voltages $V_{D C 1}$ for the first band and $V_{D C 2}$ for the second band.

TABLE I

Measured Results of THE DuAL-BAnd Tunable Active Filter

\begin{tabular}{ccc}
\hline \hline & Low-frequency band & High-frequency band \\
\hline $\begin{array}{c}\text { Tunable frequency range } \\
\text { (ripple }<3-\mathrm{dB})\end{array}$ & $698-831 \mathrm{MHz}$ & $1630-1730 \mathrm{MHz}$ \\
\hline Control voltage & $0-26 \mathrm{~V}$ & $6-26 \mathrm{~V}$ \\
\hline Maximum gain & $4.1-6.6 \mathrm{~dB}$ & $3.6-6.6 \mathrm{~dB}$ \\
\hline 3-dB bandwidth & $40-80 \mathrm{MHz}$ & $35-40 \mathrm{MHz}$ \\
\hline Input reflection & $<-6 \mathrm{~dB}$ & $<-7 \mathrm{~dB}$ \\
\hline Output reflection & $<-6 \mathrm{~dB}$ & $<-6 \mathrm{~dB}$ \\
\hline Noise figure & $6.8-7.6 \mathrm{~dB}$ & $7.5-8.5 \mathrm{~dB}$ \\
\hline
\end{tabular}

\section{CONCLUSION}

A novel tunable active filter design, based on two feedback lines in a first-order recursive topology has been analyzed. By properly isolating both lines and including phase shifters in the feedback sections, it is possible to independently tune both center frequencies. A manufactured prototype has validated the theoretical principles. Finally, it is remarked that this idea can be potentially applied for any arbitrary number of bands.

\section{REFERENCES}

[1] C. Rauscher, "Microwave active filters based on transversal and recursive principles," IEEE Trans. Microw. Theory Tech., vol. MTT-33, no. 12, pp. 1350-1360, Dec. 1985.

[2] H. Ezzedine, L. Billonnet, B. Jarry, and P. Guillon, "Optimization of noise performance for various topologies of planar microwave active filters using noise wave techniques," IEEE Trans. Microw. Theory Tech., vol. 46, no. 12, pp. 2484-2492, Dec. 1998.

[3] M. Delmond, L. Billonnet, B. Jarry, and P. Guillon, "Microwave tunable active filter design in MMIC technology using recursive concepts," in Proc. Microw. Millim. Wave Monol. Circ. Symp., May 1995, pp. $105-108$.

[4] O. Garcia-Perez, A. Garcia-Lamperez, V. Gonzalez-Posadas, M. Salazar-Palma, and D. Segovia-Vargas, "Dual-band recursive active filters with composite right/left-handed transmission lines," IEEE. Trans. Microw. Theory Tech., vol. 57, no. 5, pp. 1180-1187, May 2009.

[5] O. Garcia-Perez, L. E. Garcia-Munoz, D. Segovia-Vargas, and V. Gonzalez-Posadas, "Multiple order dual band active ring filters with composite right/left-handed cells," Progr. Electromag. Res., vol. PIER 104, pp. 201-219, May 2010.

[6] A. Lai, T. Itoh, and C. Caloz, "Composite right/left-handed transmission line metamaterials," IEEE Microw. Mag., vol. 5, no. 3, pp. 34-50, Sep. 2004

[7] H. Kim, A. B. Kozyrev, A. Karbassi, and D. W. Weide, "Linear tunable phase shifter using a left-handed transmission line," IEEE Microw. Wireless Compon. Lett., vol. 15, no. 5, pp. 366-368, May 2005.

[8] A. A. S. Mohra, "Compact dual-band Wilkinson power divider," in Proc. 25th Nat. Radio Sci. Cont. (NRSC'08), Mar. 2008, pp. 1-7. 